\title{
Design of horizontal driving machine testing platform
}

\author{
QU WEN MING ${ }^{1, a}, \mathrm{CHEN}$ QIANG QIANG ${ }^{1, \mathrm{~b}}$ and ZHU JIA DONG ${ }^{1, \mathrm{c}}$ \\ ${ }^{1}$ Hangzhou Dianzi University, Hangzhou, China

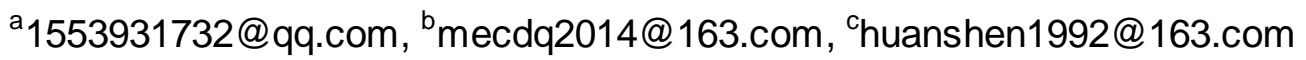

Keywords: HDM; Clamping devices; Testing platform

\section{Abstract.}

Horizontal driving machine (HDM) will directly affect the moderating effect of automobile seat, it is necessary to carry out quality inspection. This paper first puts forward the overall design of HDM test platform, then clamping devices are designed and a complete testing platform model is build.

\section{Introduction}

HDM is an important part of automobile seat to realize the automatic adjustment and the quality detection is the last link in the process of production. Quality detection of HDM can effectively reduce their failure rate. Aiming at HDM's structure, specially designed clamping devices are devised to fixed components on the platform. Cooperate with the vibration signals of the HDM at run time collected by vibration sensor: product's quality can be detected.

\section{The overall structure of testing platform}

The HDM's structure as shown in Figure 1, experimental platform is driven by rotated connecting rods which driving by the motor, the connecting rod then drive the worm in gearbox , the rotated worm then drive the worm gear, and fixed the screw, the gearbox can be moved back and forth on the screw.

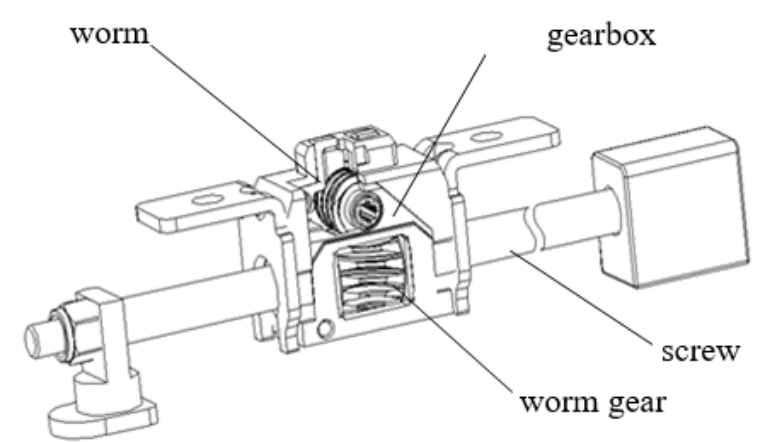

Fig. 1 The HDM's structure

Testing requirements. According to the contents need to study of experimental platform, the following two cases must be considered: the vibration of the gearbox when the motor drives the gearbox idling, gearbox not run on the screw; the vibration of the assembly when motor driven in gearbox, gear box component running back and forth on the screw.

Drive motor layout scheme design. In order to keep the gearbox run with motor, motor and gear box need to be mounted on a thin plate at the same time, and then fixed the plate on the guide.

Gearbox fixed scheme design. HDM consists of gearbox and screw, according to the contour and size of the gearbox, appropriate gearbox testing grip which composed of positioning device and clamping device should be designed.

Screw clamp scheme design. According to the layout of the motor, the screw should be fixed when the HDM experimental platform tests the vibration and the gearbox run back and forth on the screw. Quick-clamping device is selected to fix the screw according to the literature data. Through the quick clamp lever movement, the force is transmitted to the clamp pressing bar, finally screw is fixed to prevent rotation during testing through screw bearing and compaction device. 
The slider fixed scheme design. The HDM experiment platform still need to detect the vibration when motor drive gearbox individually, gearbox is not run back and forth on the screw. In the case of detection of gearbox vibration alone, because the rotation of the drive motor and gear box, the slider will move on the guide rail. Therefore, the slider must be fixed on the rail when the experimental platform tests gearbox vibration independently, so we need to design a clamp to fix slider.

Electrical control scheme design. In order to detect vibration of the gearbox better, the gearbox needs to move back and forth on he screw. Therefore, in order to give a reverse voltage to motor makes the gearbox move back when the gearbox move to the endpoint location, limit switches are needed.

\section{The testing platform grip design}

Gearbox grip design. Gearbox grip consists of positioning device and clamping device, the two parts should be fixed to the thin plate in order to fixed gearbox when detecting vibration of the gearbox. For the convenience of installation, bolts are used to fix grip. Grip's specific shape as shown in Figure 2.

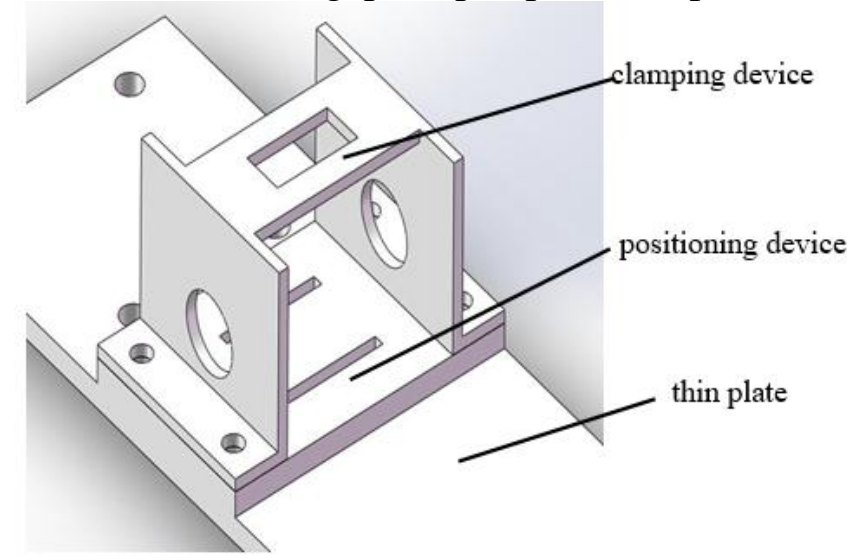

Fig.2 Gearbox grip

Motor grip design. Motor grip containing the motor mounting and motor fixture two parts, in order to make motor fixed on the plate better, we need to drilled in the motor fixture, motor plate and thin plate. The motor finally fixed on the plate by bolts, as shown in Figure 3.

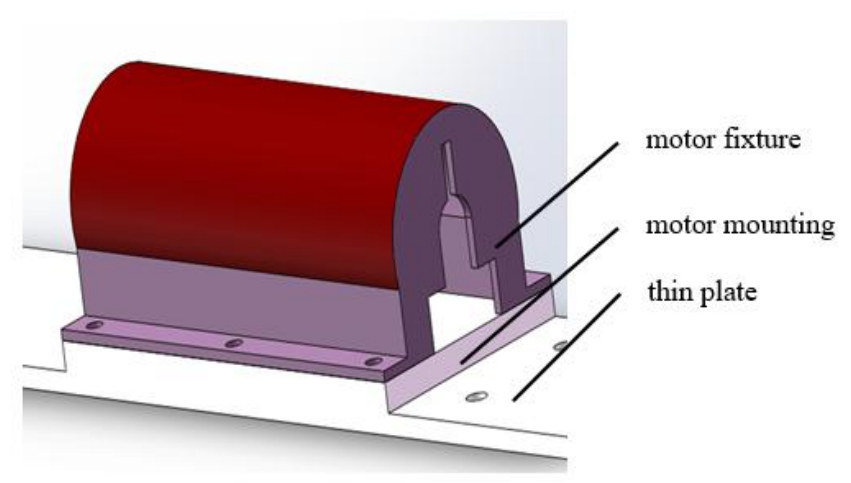

Fig.3 Motor grip

Screw fixture design. Screw fixture consists of screw bearings and pressing devices, screw bearing is made up of cuboids support block with a round hole on the top, pressing device hold the screw by the quick clamp and pressing block, the overall structure is shown in Figure 4.And the level of the pressing device can be adjusted through the nut on the pressing device, so it can hole the screw better. 


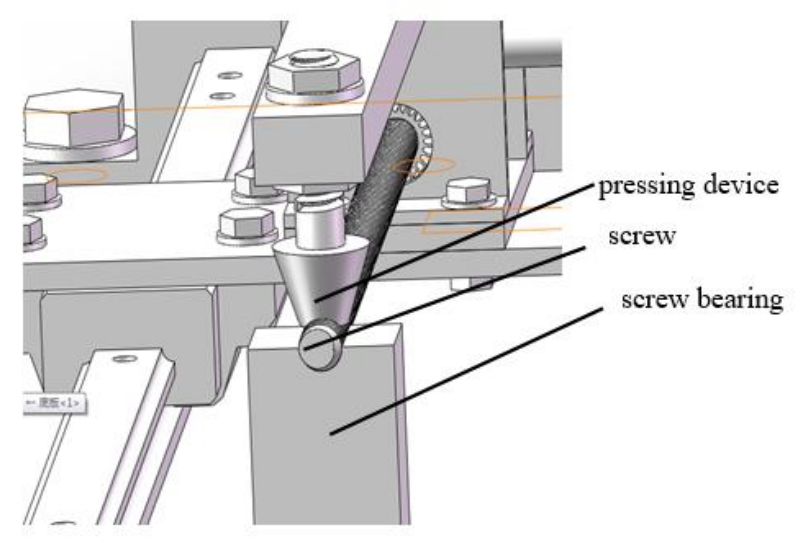

Fig.4 Screw fixture

The slider fixture design. In order to make the slider fixing device can be fixed on the linear rolling guide rail, we drilled two threaded holes on the slide block fixtures and linear guide rail according to their size and fixed via the flat cross screw. The overall structure of fixed see as shown in Figure 5

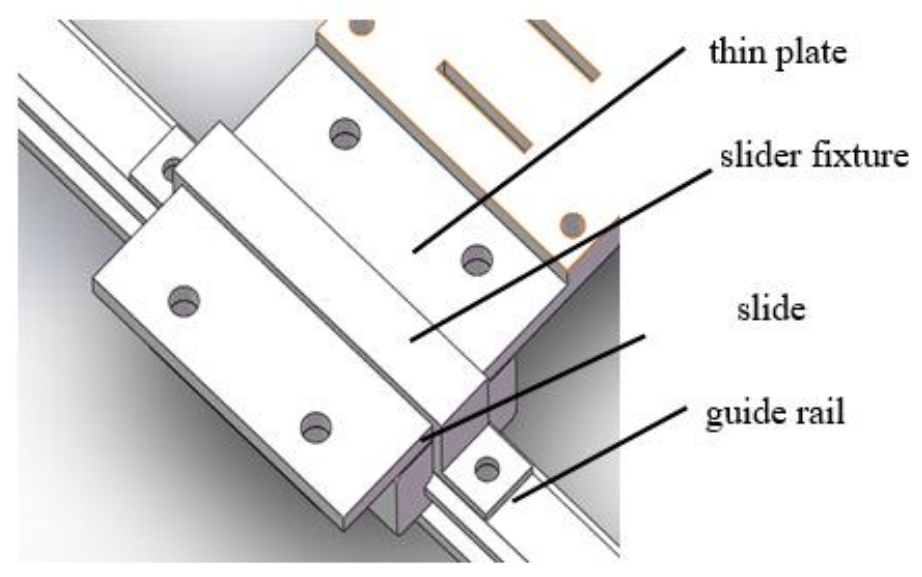

Fig. 5 Slider fixture overall structure

The travel limit switch fixture design.In order to fix the travel limit switch on the floor, we need to drill two threaded holes according to the screw holes of the limit switch at fixed position on the floor. According to rail and plate size, we choose flat cross screw to attach the base to the rail above. Thus the installation of the limit switch is ensured, limit switch and the floor can be close together to increase the strength. The overall structure shown in Figure 6.

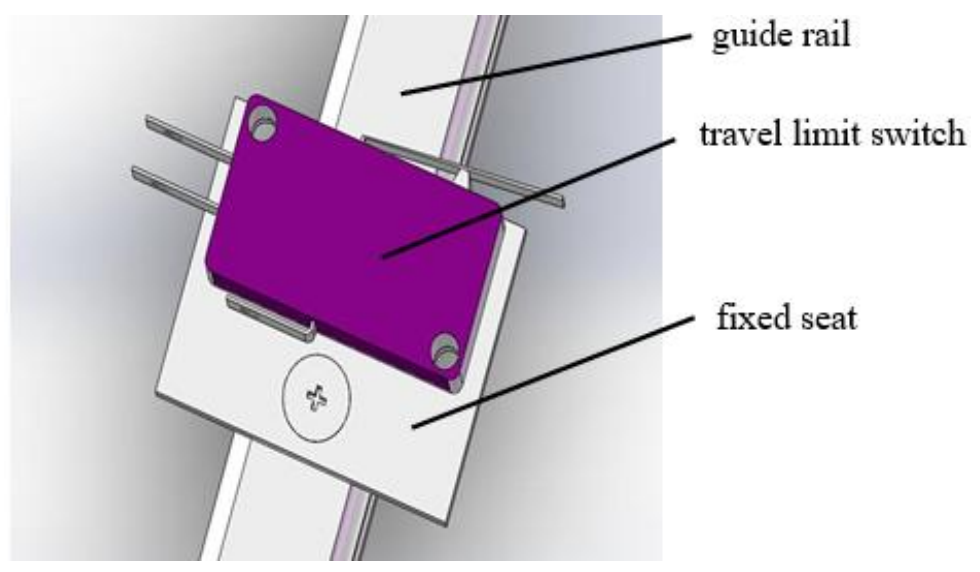

Fig.6 The travel limit switch fixture overall structure

Experimental platform overall structure. The structures of the HDM test platform as shown in Figure 7:1 for the linear guide deputy; 2 for fixed bearing; 3 pressing devices; 4 for quick clamp; 5 for pressing block 5; 6 for gearbox; 7 for thin plate; 8 for screw bearing; 9 for drive motor; 10 for travel limit switch. 


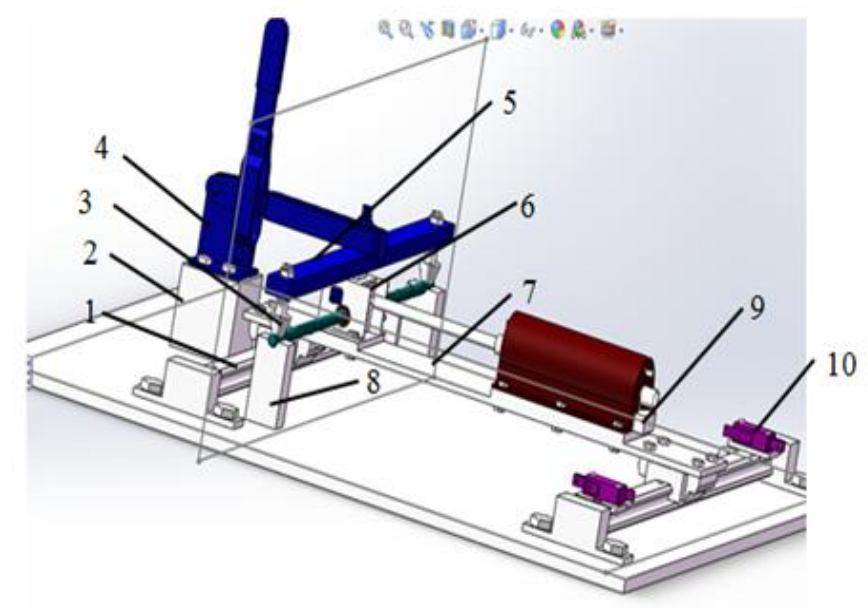

Fig. 7 Horizontal driving machine test platform

\section{VIBRATION SENSOR}

Vibration sensor is an important component parts during the detection process, its primary function is to bring the needed measurements of mechanical quantity collected and transformed, becomes propo- rtional electrical signal with it. From this we can know, vibration sensor is a kind of electromechan- ical conversion device.

Selection of sensor. Due to the HDM's gearbox of small volume and light weight, it can be thought of as a vibration system and the vibration system has a distinct effect on articulation in the $8 \mathrm{kHz}$ of the signal. But with the increase of sampling frequency, the sensitivity of the sensor is reduced, and the HDM gearbox vibration signal is weak, the useful signals are easy to be interference during the test. Considering the above factors, we choose the BK Company's 4513-002 vibration sensor. BK company dedicated cable is also used to reduce the interference, so it can get the HDM's vibration signals better and prevent interference caused by external signal.

Upon detecting the HDM's gearbox vibration, the sensor can obtain vibration signal when motor drive gearbox and convert into electrical signals, then amplified by the electronic circuit, the resultant power can be measured. Finally, by calculation, we can obtain mechanical measured quantity. Accord-ing to the amount of mechanical vibration measured by the sensor, we can determine the HDM's gearbox is qualified or not.

The installation of vibration sensor. As the general vibration sensor is relatively small when compared with the gearbox, and considering BK4513-002 small size and light weight, we choose bonded it on the gearbox fixture directly.

\section{Summary}

This paper first analyzes the overall structure of the HDM testing platform, then presents specific design methods of the gearbox grip motor grip and screw fixture, and finally a complete HDM test platform is built. Combined with the signal collected by vibra- tion sensor, we will be able to HDM's quality test-ing.

\section{References}

[1] Xu Pingjun. Horizontal driving machine (patent number: ZL03256322.1). The People's Republic of China State intellectual property office, 2004.8.

[2] Xu Pingjun, LiWei, MA Jian. A compact horizontal driving machine (patent number: ZL03256322.1.), the People's Republic of China State intellectual property office, 2007.9.

[3] Zhang Fu. Travel switches structure application [J]. China new technology and new products, 2012, (24): 76-77. 